\title{
Pascal Brioist, Léonard de Vinci, homme de guerre
}

Paris, Alma éditeur, 2013

\section{Pascal Dubourg-Glatigny}

\section{(2) OpenEdition} Journals

Édition électronique

URL : http://journals.openedition.org/artefact/546

DOI : $10.4000 /$ artefact.546

ISSN : 2606-9245

Éditeur :

Association Artefact. Techniques histoire et sciences humaines, Presses universitaires du Midi

\section{Édition imprimée}

Date de publication : 1 octobre 2016

Pagination : 411-413

ISBN : 978-2-7535-5174-9

ISSN : 2273-0753

\section{Référence électronique}

Pascal Dubourg-Glatigny, "Pascal Brioist, Léonard de Vinci, homme de guerre », Artefact [En ligne], 4|

2016, mis en ligne le 07 juillet 2017, consulté le 24 septembre 2020. URL : http://

journals.openedition.org/artefact/546; DOI : https://doi.org/10.4000/artefact.546

Ce document a été généré automatiquement le 24 septembre 2020.

\section{cc) () $\odot$}

Artefact, Techniques, histoire et sciences humaines est mise à disposition selon les termes de la Licence Creative Commons Attribution - Pas d'Utilisation Commerciale - Pas de Modification 4.0 International. 


\section{Pascal Brioist, Léonard de Vinci, homme de guerre}

Paris, Alma éditeur, 2013

Pascal Dubourg-Glatigny

\section{RÉFÉRENCE}

Pascal Brioist, Léonard de Vinci, homme de guerre, Paris, Alma éditeur, 2013, 357 p.

1 «La guerre est le seul art, pour qui commande avec compétence, capable non seulement de maintenir les princes dans leur condition mais aussi d'élever les hommes de petite extraction et de fortune privée à des niveaux supérieurs et à de grands honneurs ${ }^{1}$. » Si Niccolò Mutoni reprenait à Machiavel l'idée de l'ascension sociale par le métier des armes, il y ajoutait cependant l'idée que la guerre était le seul art pouvant y conduire. Aurions-nous oublié l'importance de la guerre dans l'histoire de la Renaissance? Que ces perpétuels conflits constituèrent le quotidien d'une grande partie de l'Europe ? Que la violence physique fut le fondement des rapports humains et sociaux? Certes, l'idée de l'humanisme l'emporte dans notre imaginaire sur un « ensauvagement » des esprits, pour reprendre le mot de Pascal Brioist, qui réduirait à néant une opération culturelle d'un extraordinaire raffinement et d'une grande complexité. La figure historique de Léonard même n'a-t-elle pas été victime de cette opération de gommage de la réalité ? Ainsi, l'ouvrage Léonard de Vinci, homme de guerre s'oppose-t-il à l'image artificielle mais confortable d'un savant et peintre quintessencié, désincarné de la réalité sanguinaire et frénétique qui l'entourait et l'accompagnait.

2 Le modèle choisi par Pascal Brioist relève du segment biographique lui permettant de suivre chronologiquement les interactions entre le phénomène militaire et les autres activités de Léonard. S'il déclare se situer dans le sillage de Paul Strathern, qui avait esquissé des portraits parfois un peu chétifs des grands hommes que Léonard avait côtoyés et servis ${ }^{2}$, et de Patrick Boucheron, qui avait étudié avec une plus grande précision le segment politique de la carrière léonardienne ${ }^{3}$, Brioist construit en 
revanche une figure historique qui n'est pas celle d'un stratège et encore moins d'un guerrier, mais plutôt celle d'un ingénieur capable de faire de l'expérience inéluctable des armes et des forteresses une ressource pour nourrir ses intérêts intellectuels plus personnels. C'est peu dire que le privilège de cette biographie est de parvenir à se détacher de l'action immédiate et de l'anecdote. Elle met en lumière les perspectives d'une carrière d'inventeur qui s'ouvrent constamment dans une multitude de directions, lui conférant la richesse exceptionnelle qu'on lui connaît. L'auteur n'en tente pas moins avec prudence de nombreux micro-rapprochements entre les événements édifiants vécus par Léonard et les instructions qu'il consigne dans ses cahiers de manière plus théorique.

3 Ce livre nous montre combien le métier des armes, comme tant d'autres arts mécaniques sans doute, peut se révéler le creuset d'une infinité d'expériences, pourvu que l'ingegno y pourvoie : la mécanique spéculative et pratique, la qualité des métaux et leur fusion, les formes des instruments de combat... Mais l'arme y est décrite dans son contexte, ce que les musées nous cachent aujourd'hui et que peu d'historiens, souvent plus attachés à une lecture patrimoniale, parviennent à montrer : l'arme n'est que l'un des instruments d'une tactique de conquête fulgurante ou de défense de position. La stratégie rejoint alors la géographie et l'architecture. Elle déploie dans l'espace l'artefact si ingénieusement élaboré. C'est bien cette conception, peut-être propre à Léonard ou plus marquée dans son approche, que ce livre nous dévoile : la capacité à envisager la moindre opération mécanique dans les conséquences - parfois difficilement prévisibles - de son usage. Il n'y a pas de limite à cette sorte de prévoyance : lorsque Léonard projette une architecture civile, il prend en considération les conséquences militaires de ses actes.

4 L'esprit d'ingénieur est ici bien développé et s'oppose à la notion, assez anachronique, "d'art technologique ", récemment proposée dans un ouvrage collectif 4 . La capacité pratique à passer de l'idée à l'objet est proche des positions développées par Romano Nanni, rassemblées dans son dernier ouvrage ${ }^{5}$. La mission de l'ingénieur correspond certes à des compétences mais met en exergue une disposition d'esprit, une disponibilité à la mobilité intellectuelle et une indifférence pour le caractère disciplinaire des activités humaines. Cette fonction, car il s'agit là plus d'une fonction que d'une profession, induit des circulations entre le savoir issu de l'expérience et différents types d'applications. Il ne s'agit pas à proprement parler d'un passage entre théorie et pratique. Le mode d'élaboration intellectuel de Léonard - des notes à l'apparence parfois morcelée - en est une preuve ultérieure.

5 Si l'ouvrage ne succombe pas à l'image éculée du courtisan, il n'en pose pas moins la question de l'allégeance politique versatile des artistes et savants. La carrière de Léonard évolue depuis ses débuts, très marqués par des activités liées à la guerre, en particulier au service de César Borgia, à une époque où la connotation plus civile prévaut, alors que Louis XII le désigne comme "peintre et ingénieur ordinaire ». Cette narration traditionnelle est ici modulée par la continuité des préoccupations. Léonard aurait-il intégré son rapport à la guerre dans une représentation qui cristallise un vécu personnel et une longue carrière d'expérimentation? Pascal Brioist défend cette position dans une analyse des conditions d'élaboration de la fresque perdue de la bataille d'Anghiari. Ces pages substituent l'impossible description par une contextualisation serrée. Une proposition assez différente de celle suivie par Carlo Vecce dans sa lecture vincienne de 2011 qui s'en tenait à un commentaire textuel ${ }^{6}$. 
6 Si la littérature sur Léonard ne tarit pas et qu'il devient malaisé de se distinguer dans un panorama désormais foisonnant, l'approche spécifique choisie par l'auteur se nourrit d'une maturité certaine des sources. Les manuscrits de Léonard sont accessibles sur le réseau et le chercheur peut y explorer les chemins privilégiés par le truchement d'outils sophistiqués et efficaces, y compris la transcription des textes. L'entreprise du glossario leonardiano se poursuit, offrant les meilleurs instruments lexicographiques dans le domaine habituellement négligé de la langue technique de la Renaissance ${ }^{7}$. Nul doute que l'ouvrage de Pascal Brioist, offrant un cadrage nouveau sur cette figure, en adéquation avec le quotidien hoplomachique de la Renaissance, nous aide à mieux comprendre la complexité et l'originalité du parcours d'un ingénieur au sens le plus puissant du terme.

\section{NOTES}

1. POLYEN (II ${ }^{\mathrm{e}}$ après J.-C.), Stratagemi dell'arte della guerra, traduit du grec en italien par N. Muttoni, Venise, 1551, épître dédicatoire.

2. Paul STRATHERN, The Artist, the Philosopher and the Warrior, New York, London, Jonathan Cape, 2009.

3. Patrick BOUCHERON, Léonard et Machiavel, Lagrasse, Verdier, 2013.

4. Agostino catalano, Rossano Pazzagli (éd.), Arte e tecnica in Leonardo ingegnere, Ariccia, Aracne, 2014.

5. Romano NANNI, Leonardo e le arti meccaniche, Milan, Skira, 2013.

6. Carlo VECCE, Le Battaglie di Leonardo, Florence, Giunti, 2012.

7. La seconde et dernière en date de ces publications est le très utile ouvrage de Margherita QUAGLINo, Glossario leonardiano. Nomenclatura dell'ottica e della prospettiva nei Codici di Francia, Florence, Olschki, 2014.

\section{AUTEURS}

\section{PASCAL DUBOURG-GLATIGNY}

CNRS-Centre Alexandre Koyré 